\title{
Exploration of grid scheme for Frisch-grid ionization chamber
}

\author{
Meng $\mathbf{L i}^{1,2, \mathrm{a}} \mathbb{1}$, Yuansheng Yang ${ }^{1,2}$, Peng Ma ${ }^{1,2}$, Junwei Zhang ${ }^{3}$, Zhi Qin ${ }^{4}$, Xianglun Wei ${ }^{1}$, Chaoyuan Xiao ${ }^{5}$, \\ Tianli Qiu ${ }^{1,2}$, Zhoubo $\mathbf{H e}^{1,2}$, Zhixuan $\mathbf{H e}^{5}$, Chengui $\mathbf{L u}^{1,2}$, Rongjiang $\mathbf{H u}^{1,2}$, Herun Yang ${ }^{1,2, b}$, Yongde Fang ${ }^{1,2}$, \\ Zhigang Xiao ${ }^{4}$, Hongwei Wang ${ }^{2,6}$, Yikai Hou ${ }^{5}$, Limin Duan ${ }^{1,2}$, Hooi Jin Ong ${ }^{1,2,7,8}$ \\ ${ }^{1}$ Institute of Modern Physics, Chinese Academy of Sciences, Lanzhou 730000, China \\ ${ }^{2}$ School of Nuclear Science and Technology, University of Chinese Academy of Sciences, Beijing 100049, China \\ ${ }^{3}$ North China University of Water Resources and Electric Power, Zhengzhou 450000, China \\ ${ }^{4}$ Department of Physics, Tsinghua University, Beijing 100084, China \\ ${ }^{5}$ School of Nuclear Science and Technology, Lanzhou University, Lanzhou 730000, China \\ ${ }^{6}$ Shanghai Institute of Applied Physics, Chinese Academy of Sciences, Shanghai 201800, China \\ 7 Joint Department for Nuclear Physics, Lanzhou University and Institute of Modern Physics, Chinese Academy of Sciences, Lanzhou 730000, \\ China \\ ${ }^{8}$ Research Center for Nuclear Physics, Osaka University, Ibaraki, Osaka 567-0047, Japan
}

Received: 31 August 2021 / Accepted: 24 November 2021 / Published online: 10 December 2021

(C) The Author(s) 2021

\begin{abstract}
A new Frisch-grid ionization chamber has been built to explore the appropriate choice of Frisch-grid. Detailed studies of the relationship between grid geometries and detector performance have been performed with an ${ }^{241} \mathrm{Am}$ source. This paper describes and compares the energy resolution of ionization chambers with parallel-wire and mesh grids of different grid parameters. Some specific recommendations for grid selection are provided based on the data currently available. To obtain optimal energy resolution, the operating voltage of the chamber must satisfy the condition of minimum electron collection on the grid with distinct geometries and parameters, respectively. Since there is no established theory applicable to both types of grids, we have devised a careful simulation procedure incorporating the COMSOL and Garfield++ codes to search for the conditions of the minimum electron collection on the grid. The simulation results fit the experimental data well, suggesting that this simulation method successfully predicts the suitable voltage setting when using a mesh grid or parallel wires grid as the shielding electrode.
\end{abstract}

\section{Introduction}

The Frisch-grid ionization chamber (FGIC) is widely used in nuclear physics experiments to measure the energy, angle or mass of an incident ion beam or charged particles emitted during nuclear reactions $[1,2]$. The FGIC concept, first

\footnotetext{
a e-mail: limeng017@impcas.ac.cn

be-mail: yanghr@impcas.ac.cn (corresponding author)
}

introduced by Frisch [3], was developed from the parallelplate ionization chamber. FGIC consists of three parallel electrodes: a cathode, an anode and a grid. The metal grid located between the cathode and the anode divides the gas volume into the electron drift region and the electron collection region. Only when the electrons pass through the grid can they begin to generate the signal induction on the anode, thus, effectively eliminating the dependence of the anode induction signal on the ionization position and orientation in the chamber. Therefore, an advantage of FGIC is that the pulse height of the anode is only related to the energy deposit of charged particles. In addition to the accurate energy measurement, another advantage of FGIC is that the emission angles of ionizing particles can be obtained by analyzing the grid and anode signal [4].

An ideal grid shields the moving electrons in the electron drift region completely, preventing them from inducing a signal on the anode. In reality, however, such condition cannot be realized, and a weak charge induction still exists on the anode. This imperfect shielding is known as the grid inefficiency. Bunemann et al. proposed the mirror-charge theorem, and provided a formula to calculate the grid inefficiency $\sigma$ [5]. However, it is only applied to the parallel-wire grid, and there is no theoretical formula for the other grid geometries, such as meshes. Göök et al. adopted the Shockley-Ramo theorem [6,7] to quantify the parameter $\sigma$ by introducing an experimental method that determines the grid-inefficiency correction factor [8]. The experimental results and the calculated values are in good agreement for both types of grids. A paper by Al-Adili et al. confirmed the method discussed in 
Ref. [8], and investigated the uncertainty in the determination of the grid-inefficiency correction factor [9]. Both works provided complementary understanding to the charge induction shielding of the grid.

In addition, an ideal grid does not intercept the drifting electrons resulting in the loss of collected charges on the anode. A large number of experimental findings demonstrated that when the electric field between the cathode-grid region $E_{\mathrm{cg}}$ and the electrical field between anode-grid region $E_{\text {ag }}$ exceeds a certain ratio, the loss of electrons to the grid is minimized. The least ratio of $E_{\mathrm{ag}}$ to $E_{\mathrm{cg}}$ is primarily relevant to the structural parameters of the chamber. It is described by

$R=\frac{E a g}{E c g} \geq R_{\min }=\frac{1+\rho}{1-\rho}, \rho=\frac{2 \pi r}{d}$,

where $r$ and $d$ are the grid-wire radius and spacing, respectively. This formula also originates from the theoretical derivation based on the parallel-wire grid, and does not take electron diffusion into account. In reality, the theoretical minimum field ratio is lower than the experimental value [10].

For optimal operation of FGIC, the grid is desired to be transparent to electrons while providing complete electrostatic shielding, simultaneously. Both characteristics of the grid have opposite effects on the pulse height of anode signal, and affect the energy resolution of FGIC. Hence, we need to explore grid parameters that provide the best solution in term of the energy resolution. In this work, the effect of different grid types and grid parameters on the energy resolution was investigated with an ${ }^{241} \mathrm{Am}$ source. A suitable FGIC operating voltage must consider the electron transmission across the grid. In view of the incomplete theory in determining the minimum electric field ratios $R_{\text {min }}$ of the grid so far, we also applied the finite element method combined with a Monte Carlo simulation to describe the electron transmission across the grid to determine the $R_{\min }$ value.

\section{Construction and operation}

\subsection{Detection system}

A schematic drawing of the established FGIC, and a photo of our prototype FGIC are illustrated in Fig. 1. The chamber consists of the drift region (cathode to grid), the Frisch grid, and the collection region (grid to anode). The cathode and anode are made of printed circuit board coppered on one side with an active area of $150 \times 150 \mathrm{~mm}^{2}$. The cathode has several small holes of $0.7 \mathrm{~mm}$ diameter at its center to collimate alpha particles. The grid is an electrode plate made of printed circuit board frame spanned with a gold-plated tungsten wire or gold-plated $\mathrm{Be}-\mathrm{Cu}$ wire. The cathode-grid and anode-grid distances were set to $112 \mathrm{~mm}$ and $22 \mathrm{~mm}$, respectively.
To achieve high energy resolution, electric field homogeneity is required in the active region. The field cage is composed of four printed circuit boards, each with a width of $1.2 \mathrm{~mm}$ parallel electrode strip in $2 \mathrm{~mm}$ steps etched on both sides. Compared with the field cage made of metal rings and wires, it is known for its simple manufacturing process, mature technology, and high precision. All the parallel electrode strips are connected through $1 \mathrm{M} \Omega$ resistors forming a resistor chain to achieve field homogeneity.

\subsection{Working gas}

FGIC is mounted on an aluminum flange, which can be directly fixed to a stainless steel cylindrical chamber using nylon studs. The chamber has a wall thickness of $3.5 \mathrm{~mm}$, and an inner diameter of $318 \mathrm{~mm}$. The detector was operated with the P-10 gas (a gas mixture of $10 \% \mathrm{CH}_{4}$ and $90 \%$ argon), and was gas sealed throughout the measurements. At the beginning of each test, it was flushed with the P-10 gas. Over time, the quality of the counting gas may deteriorate due to the degradation of the P-10 gas, which is characterized by the gradual drift of the signal pulse height. Therefore, the filled gas should be changed periodically.

According to the calculation using the Stopping and Range of Ions in Matter (SRIM) code [11], the projected range of a $5.5-\mathrm{MeV}$ alpha particle is around $5 \mathrm{~cm}$ in the $\mathrm{P}-10$ gas at 1 atm. The projected range reflects the stopping power of the charged particles in the gas. Because the detection principle of FGIC requires that all charged particles are stopped in the cathode-grid region, to prevent the alpha particles from leaving the cathode-grid region without depositing their full energy, the minimum pressure of the P-10 gas should be above 500 mbar. In this work, measurements were performed at 550 and 1000 mbar gas pressure.

\subsection{Voltage setting}

FGIC usually operates at three voltages, with the grid at an intermediate potential. In our experiment, the grid was connected to the ground without passing through any electronic equipment. As pointed out in Ref. [12], this treatment can enhance the signal quality. Both the ionization of charged particles and the electron drift process mainly occur in the cathode-grid region. To minimize the electron capture, electrons are supposed to travel with a high drift velocity in the cathode-grid region. The electron drift velocity in the counting gas depends on the reduced electrical field $E / P$, where $E$ and $P$ represent the electric field strength and the gas pressure, respectively. For the P-10 gas with a reduced electrical field of $130 \mathrm{~V} / \mathrm{cm} / \mathrm{bar}$, the fastest electron drift velocity amounts to approximately $5.5 \mathrm{~cm} / \mu \mathrm{s}$ as deduced from the Magboltz code [13]. In addition, it can offer the most stable drift mobility with respect to the slight fluctuations in 
Fig. 1 Schematic drawing (a) and photogragh (b) of the FGIC used in this work

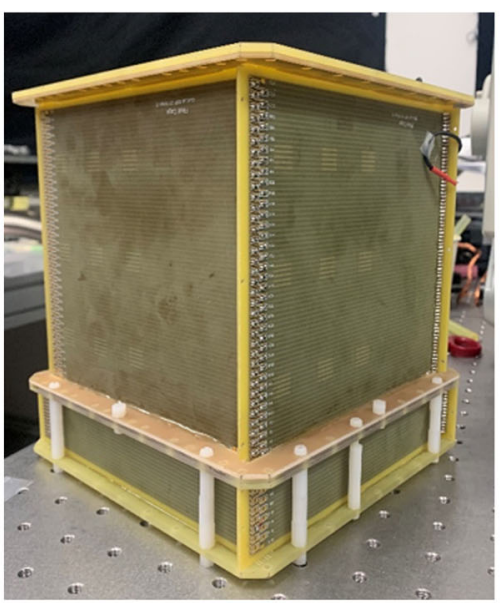

(a)

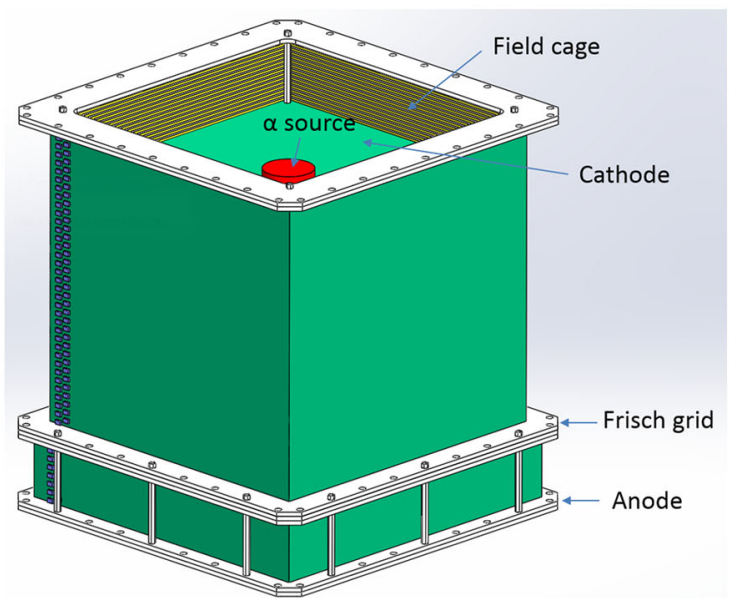

(b)

Table 1 General characteristics of the ORTEC preamplifier model 142PC and AMPTEK CoolFET preamplifier A250CF

\begin{tabular}{lllll}
\hline & Rise time & Decay time & Sensitivity & Noise \\
\hline ORTEC 142PC & $25 \mathrm{~ns}(0 \mathrm{pF})$ & $50 \mu \mathrm{s}$ & $300 \mathrm{mV} / \mathrm{MeV}(\mathrm{Si})$ & 600 electrons FWHM \\
Amptek CoolFET A250CF & $2.5 \mathrm{~ns}(0 \mathrm{pF})$ & $1 \mathrm{~ms}$ & $176 \mathrm{mV} / \mathrm{MeV}(\mathrm{Si})$ & 300 electrons FWHM \\
\hline
\end{tabular}

the inhomogeneity of the electrical field. Hence, the cathode voltage was set to $-850 \mathrm{~V}$ at $550 \mathrm{mbar}$, and $-1550 \mathrm{~V}$ at 1000 mbar, separately. The anode is on the positive potential. In order to avoid electron loss at the grid, while keeping the cathode voltage constant, the optimal anode voltage was determined by varying the ratio of $E_{\mathrm{ag}}$ to $E_{\mathrm{cg}}$.

\subsection{Electronics}

For a good energy resolution, the suppression of electronic noise is essential for FGIC. The use of the AMPTEK CoolFET preamplifier [14] with a Peltier cooled FET can significantly reduce the electronic noise [8]. In the experimental setup, the anode was directly connected to the CoolFET preamplifier with DC coupling. The anode preamplifier output was sent to the ORTEC 572A amplifier with a shaping time of $3 \mu \mathrm{s}$. The energy signal was coded on a multi-channel analyzer ORTEC 927. It should be noted that the AMPTEK preamplifier has a long decay time, which can reduce the ballistic deficit. However, the resulting pile-up of pulses should be considered in an accelerator-based test. For comparison, the conventional ORTEC 142PC preamplifier [15] with a shorter decay time was also used. Table 1 summarized general characteristics of these two preamplifiers.

Additionally, to reduce the environmental noise in the laboratory, we connected the high-voltage power supply of the detector and its subsequent electronic system through a noise
Table 2 The grid parameters of two different types of grids

\begin{tabular}{llcc}
\hline & Grid type & Wire radius $\mathrm{r}(\mathrm{mm})$ & Wire spacing $\mathrm{d}(\mathrm{mm})$ \\
\hline Grid 1 & Parallel wires & 0.025 & 0.5 \\
Grid 2 & Mesh & 0.025 & 0.5 \\
Grid 3 & Parallel wires & 0.0375 & 0.75 \\
Grid 4 & Mesh & 0.0375 & 0.75 \\
\hline
\end{tabular}

cut filter to avoid noise interference from other power supply equipment.

\section{Experimental setup}

Two different types of grid geometry were used for detailed measurements. The parallel-wire grids were made of goldplated tungsten wires. The mesh grids consisted of two sets of vertically interlaced parallel wires made from gold-plated $\mathrm{Be}-\mathrm{Cu}$ wires. The various grid parameters employed in this experiment are given in Table 2. The energy resolution of FGIC was measured with an ${ }^{241} \mathrm{Am}$ alpha source with four different grids used alternately. As mentioned earlier, the alpha source was collimated through several small holes at the center of the cathode. The reduced electric field between the cathode and the grid was kept constant during the entire experiment. The energy spectra were recorded with increasing $E_{\mathrm{ag}} / E_{\mathrm{cg}}$ settings. 


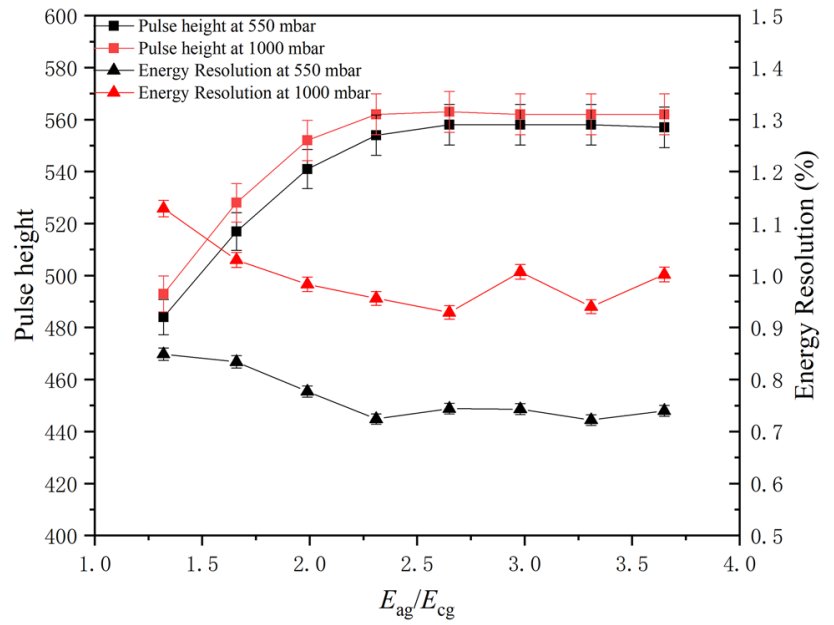

Fig. 2 The energy resolution and pulse height evolution of a series of $E_{\mathrm{ag}} / E_{\mathrm{cg}}$ in $550 \mathrm{mbar}$ and $1000 \mathrm{mbar}$. The uncertainties are dominated by statistical variation

\section{Results and discussion}

\subsection{Determination of the operation condition}

To determine the appropriate working condition of the detector, the energy resolution and pulse height as functions of the voltage setting ratio were measured at 1000 mbar and 550 mbar using Grid 1 and the AMPTEK CoolFET A250CF preamplifier. Figure 2 shows the corresponding result. Because the number of electrons captured by the grid decreases with increasing anode voltage $\left(E_{\mathrm{ag}} / E_{\mathrm{cg}}\right)$, more electrons free from the grid can be collected by the anode, thus resulting in the increase in the pulse height of the anode signal. Similarly, the signal-to-noise ratio increases, and the energy resolution improves with increasing anode voltage. The overall trend is similar for both pressures of 550 mbar and 1000 mbar. Additionally, the anode pulse height reaches the plateau region when the $R$ value exceeds 2.65 . The energy resolution also reaches almost the optimal values. However, the energy resolution is slightly better at lower pressures with smaller fluctuations.

In the following, we describe the procedures to determine the energy resolution. Figure 3 presents the energy spectrum obtained at 550 mbar P-10 gas with an ${ }^{241}$ Am source. For the nuclide ${ }^{241} \mathrm{Am}$, three major branches of the alpha decay exist:

(a) The main alpha decay branch $\left(E_{\alpha}=5.486 \mathrm{MeV}, \mathrm{BR}\right.$ (branching ratios) $=84.8 \%$ ) contributes to the main peak of the energy spectrum,

(b) The other branch $\left(E_{\alpha}=5.443 \mathrm{MeV}, \mathrm{BR}=13.1 \%\right.$ ) contributes to the low-energy tail of the energy spectrum,

(c) The third $\left(E_{\alpha}=5.545 \mathrm{MeV}, \mathrm{BR}=0.37 \%\right)$ is negligible, due to its small branching ratio and large energy interval.

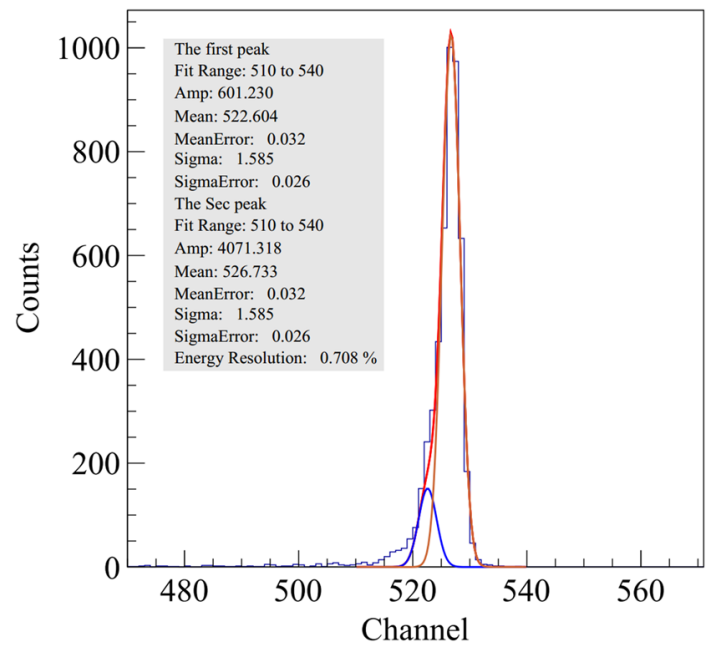

Fig. 3 Energy spectrum obtained with an ${ }^{241} \mathrm{Am}$ source. The red line (on top of the data) represents the total adjustment to the data taking into account the known alpha peak energies and branching ratios. The orange and blue lines, shown as two single Gaussians, represent the two different alpha contributions with branching ratios of $84.8 \%$ and $13.1 \%$, respectively

A double Gaussian fitting function was constructed based on the contribution of the two major branches of the alpha decay. Their relative BR and $E_{\alpha}$ were considered in defining the related parameters of the function, while the widths of the two peaks were assumed to be the same, thus reducing the number of free fitting parameters to three. The energy resolution of the chamber was calculated using the full width at half maximum (FWHM) and peak position of the main alpha decay branch with a branching ratio of $84.8 \%$. Throughout all of the measurements, we used identical methods to determine the FWHM and the peak position. The optimal energy resolution of the chamber was $0.7 \%$, as presented in Fig. 3.

\subsection{Impact of emission angle}

Due to the presence of grid inefficiency, the anode signal height remains slightly angular-dependent. Therefore, it is necessary to study the difference in energy resolution when FGIC receives ionizing particles with different emission angle. The ${ }^{241} \mathrm{Am}$ source was collimated through two different holes, with 0.5 and $0.7 \mathrm{~mm}$ diameter, leading to estimated maximum emission angles of alpha particles of $28^{\circ}$ and $39^{\circ}$, respectively. Figure $4 \mathrm{a}, \mathrm{b}$ shows the optimal energy resolution of FGIC using the two collimation holes. Both spectra were obtained by the ORTEC 142PC preamplifier. The energy resolution from the measurements with different emission angles are very close, and the resolution difference can be considered within the range of error and fluctuation. This is experimental verification of the good shielding performance of the grid we used. As intended, the impact of 


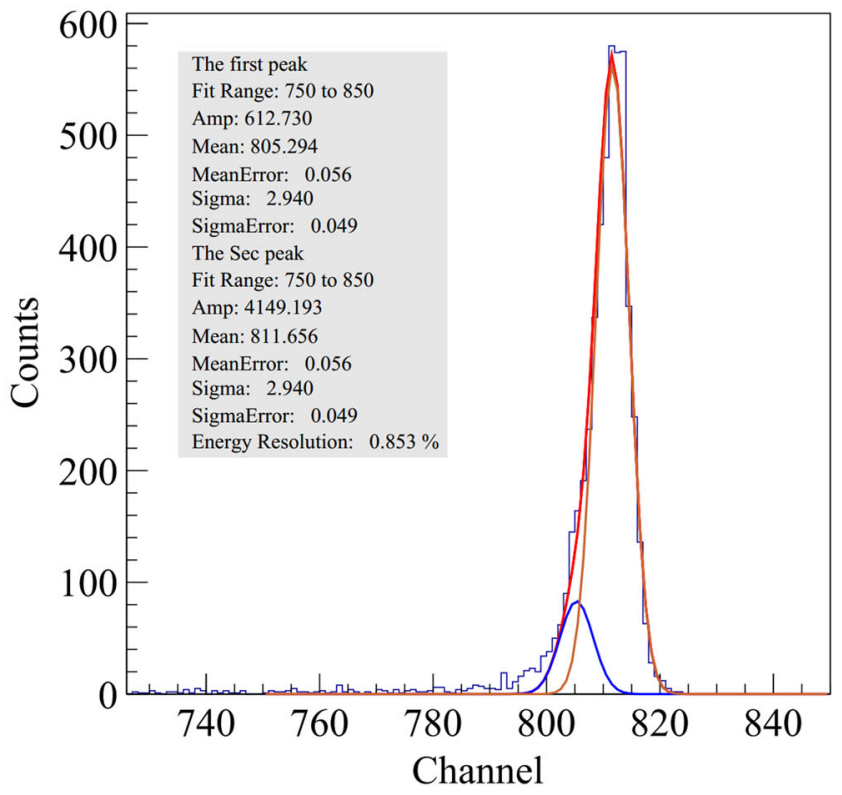

(a)

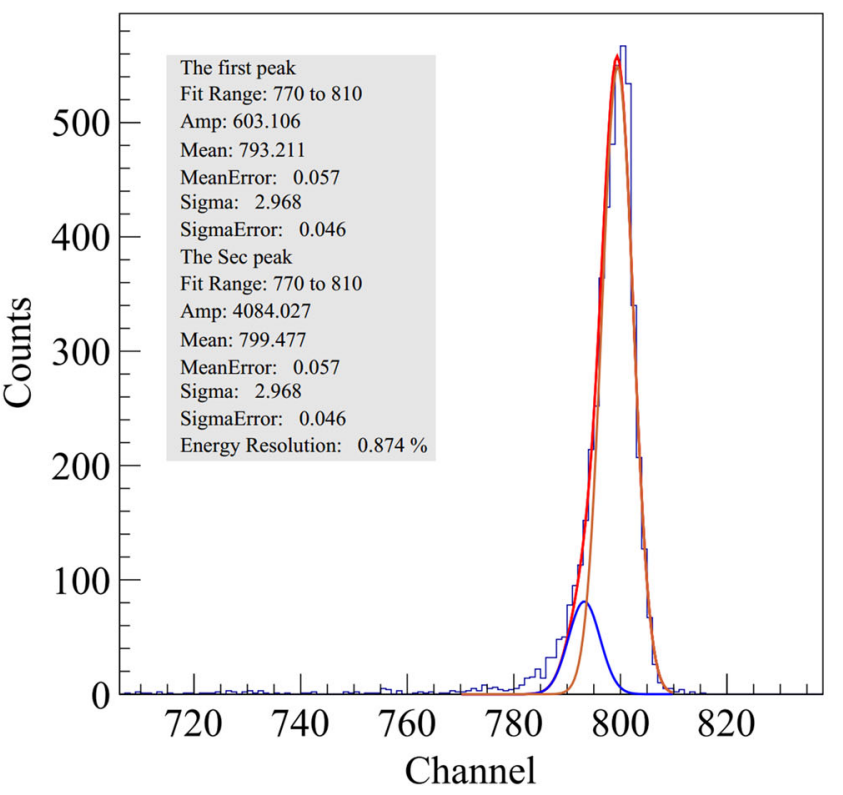

(b)

Fig. 4 The energy spectra from the two identical measurements, with 0.5 (a) and $0.7 \mathrm{~mm}$ (b) collimation holes

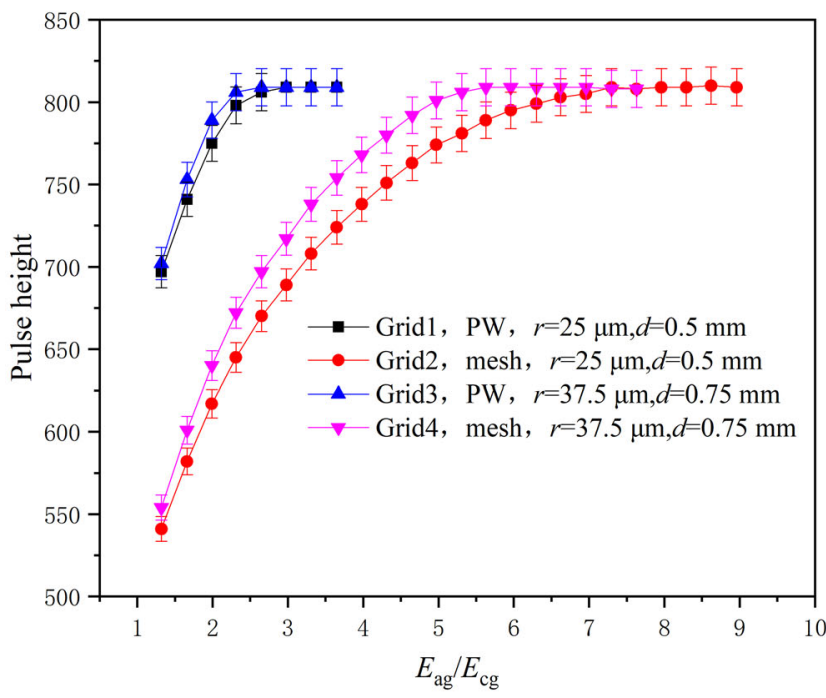

(a)

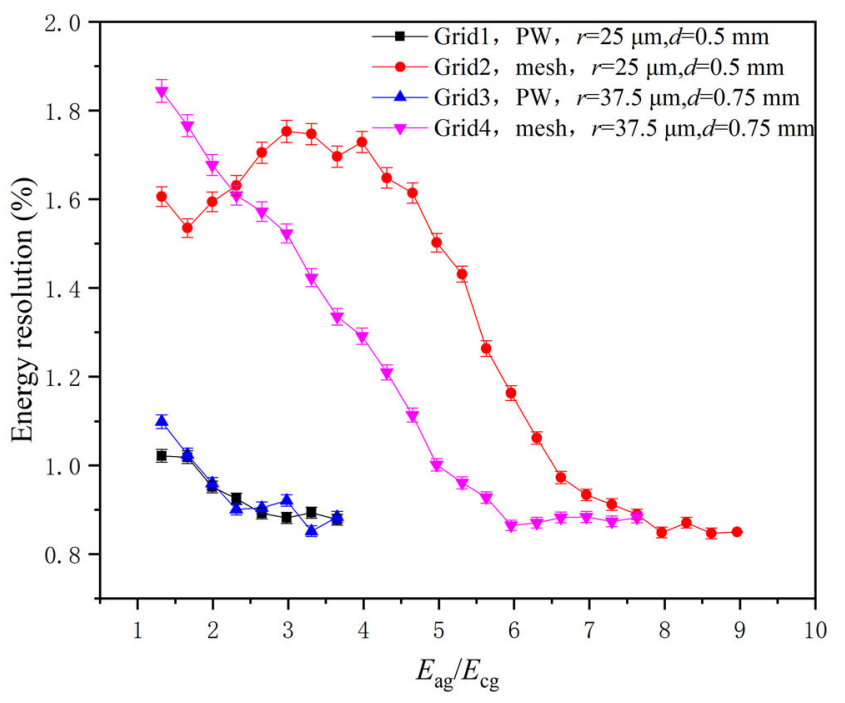

(b)

Fig. 5 Graphs showing the anode pulse height (a) and chamber resolution (b) as functions of $E_{\mathrm{ag}} / E_{\mathrm{cg}}$ when using four different grids. The different grids are denoted by different symbols. The error bars are

particle emission angle on energy resolution is negligible if the grid has good shielding.

\subsection{Comparison of different grid geometries}

Applying the above-mentioned procedures in Sect. 4.1, measurements with different grid geometry types were performed using the P-10 gas at 550 mbar. We used the ORTEC 142PC preamplifier in these measurements, while keeping other

mainly stemming from statistical varieation. PW denotes the parallel wire grid. The notation is the same as in Table 2: $r$ is the wire radius, and $d$ is the wire spacing

electronics unchanged. Figure 5 shows the results. For each grid, the anode pulse height remained stable at a similar value when exceeding respective minimum electrical field ratio, indicating that $100 \%$ electron transparency was achieved in different grid geometries. Even for the mesh grid with a very high wire density, electrons were not lost to the grid, as long as there was a sufficiently strong electric field strength in the grid-anode region to pull them down. The optimal energy resolution for the four grids are compared in Fig. 5b. Both 


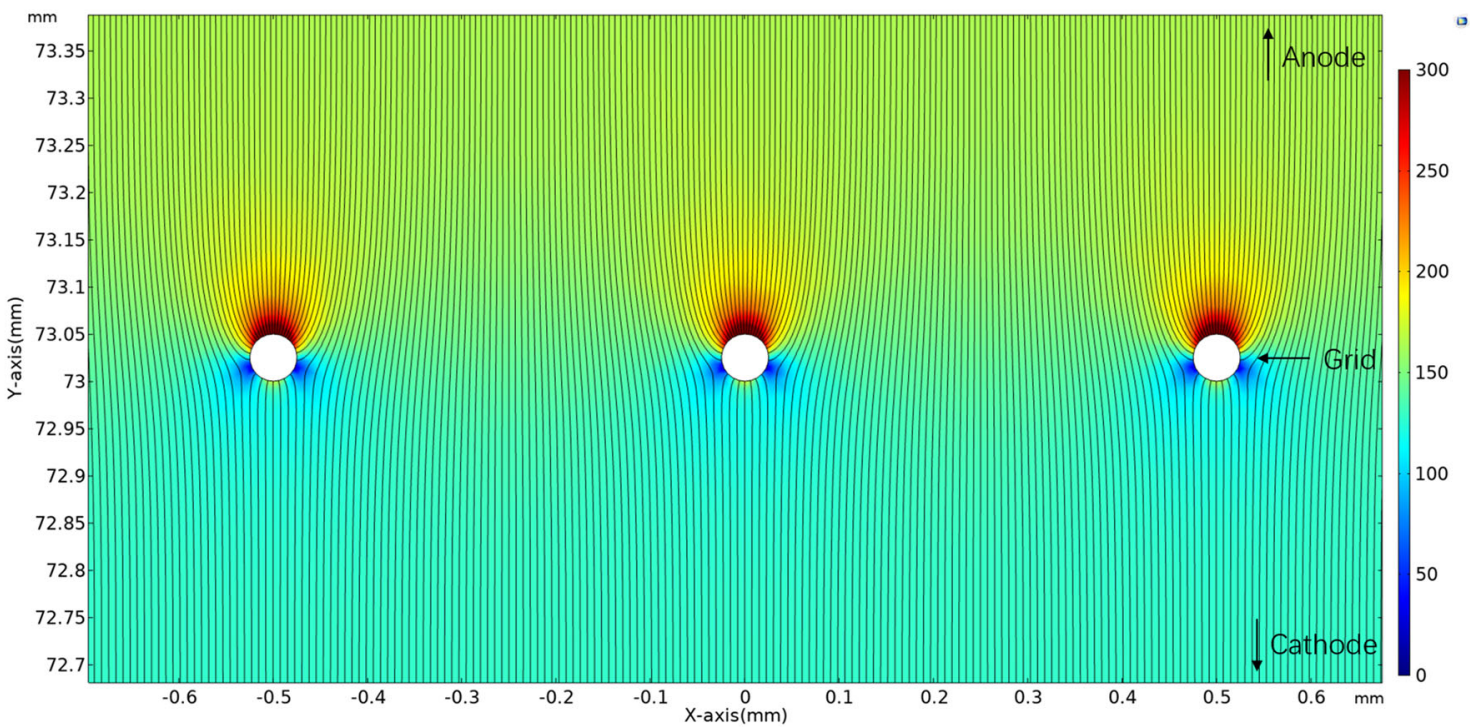

(a)

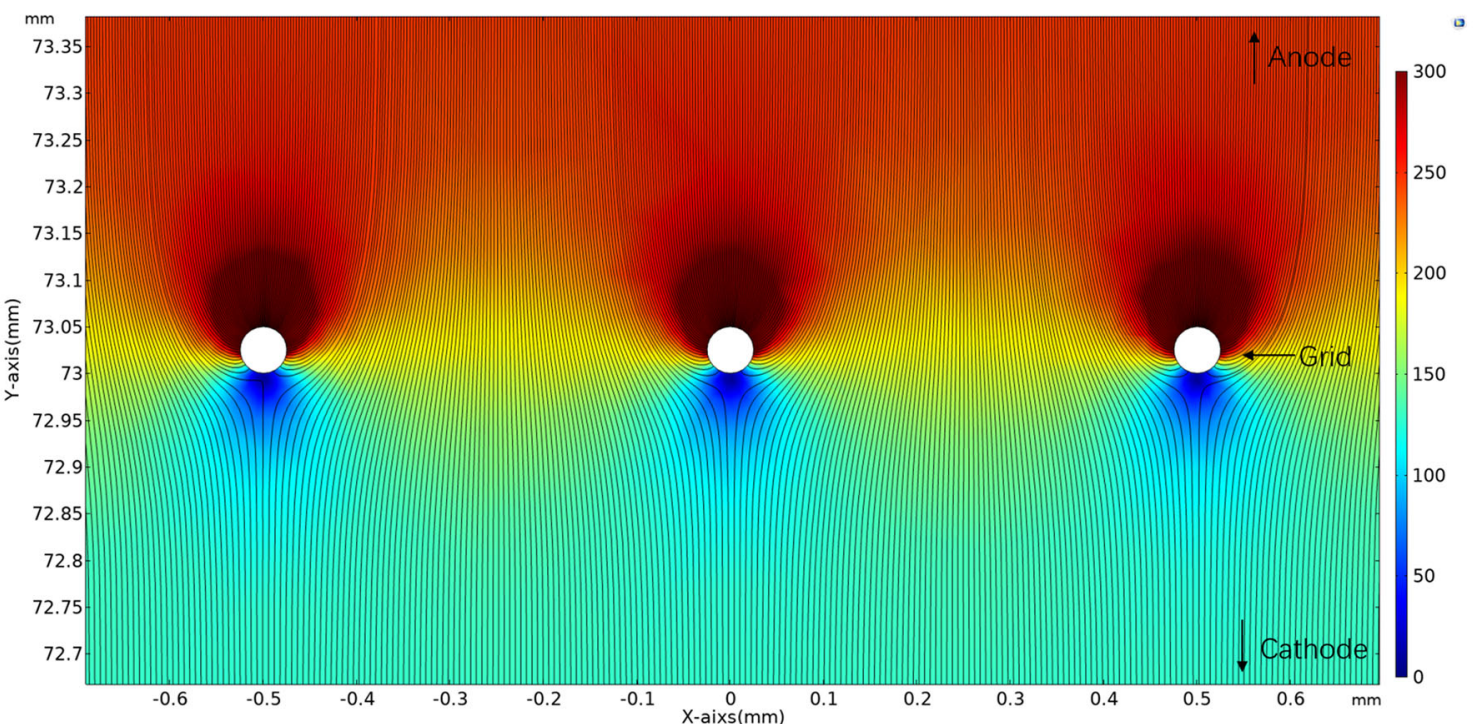

(b)

Fig. 6 The Graph on the top a shows the distribution of the electric field lines around the grid wires for a voltage setting of $R<R_{\min }$, and on the bottom $\mathbf{b}$ is for the case of $R \geq R_{\min }$

the parallel-wire and mesh grids exhibit a similar optimal energy resolution of about $0.85 \%$ after reaching $100 \%$ electron transparency.

It is worth noting that an excellent resolution was achieved by using two different grid geometries, and no improvement in resolution was observed with the use of a mesh grid from Fig. 5b. This result is inconsistent with an earlier work, which reported a better energy resolution with a mesh grid [16]. In Ref. [16], the peak position and peak width for three grid types were compared at electric field ratios of 3. While Eq. (1) is fulfilled by the parallel-wire grid, as mentioned in the
Introduction, it is invalid for the mesh grid. Hence it is reasonable to suspect that the electron transparency for the mesh grid was not $100 \%$ during their measurements, and the discrepancy may have arisen from the suboptimal conditions.

The four tested grids comprise the parallel-wire grid and mesh grid with distinct grid parameters, and hence different $\sigma$ values. The fact that the energy resolution of FGIC remains consistent when using any of the four grids, implies that they all provide sufficient electrostatic shielding. In some cases, grids with large pitches of the wires provide inadequate shielding, and the chamber resolution deteriorates with 


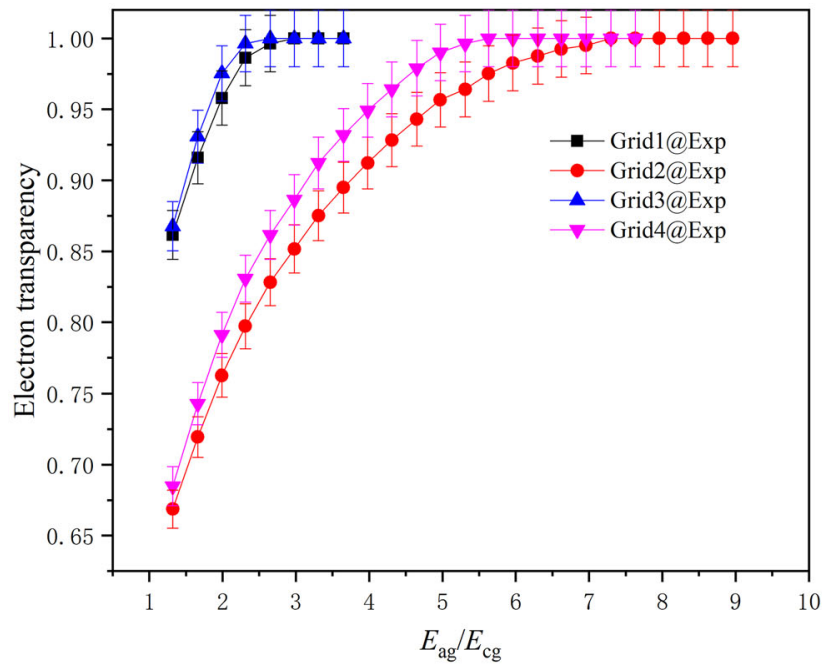

(a)

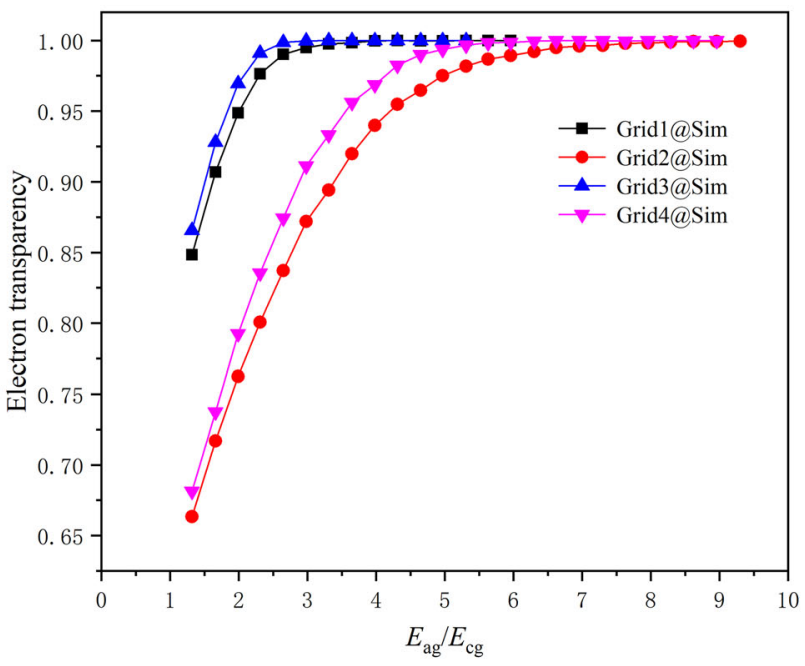

(b)

Fig. 7 Experimental (a) and simulated (b) electron transparency of different grids as a function of $E_{\mathrm{ag}} / E_{\mathrm{cg}}$

increasing wire spacing [17]. Based on the current results, we consider that there is a saturation limit for the grid parameters. For either the mesh or the parallel wire, when the grid exhibits a small enough shielding inefficiency, the influence of the grid inefficiency on the anode signal becomes negligible. In this case, any effort to reduce the $\sigma$ value by adjusting the grid parameters is futile. Such effort will only serve to increase the required electric field ratio.

\subsection{Simulation of electron transparency}

The results in Fig. 5 show that the minimum electric field $E_{\min }$ can be determined experimentally. However, when designing a detector, it is important and preferable to simulate the detector response rather than adopting the trial-anderror approach. The Garfield++ package is primarily dedicated to the simulation of the Micro Pattern Gaseous Detector [18]. It is fully capable of simulating response of ionization chamber, which mainly involves transportation process of electrons including diffusion, attachment, recombination, and drift, but excluding the electron avalanche process. The Garfield++ code can interface with various finite element programs by importing finite-element electric field maps. Compared to the simple two-dimensional modeling installed in Garfield++, finite element technique can manage complex electrode shapes, and thus can be used to construct the threedimensional detector geometry. For this purpose, we employ the COMSOL software [19].

Before interfacing COMSOL with Garfield++, the threedimensional configurations of the grid ionization chamber with Grids 1-4 installed was created with the COMSOL software. The series of voltages loaded on the model is the same as that in Fig. 5. The screenshot shown in Fig. 6 depicts the distribution of the electric field lines in the vicinity of the grid. As expected, a large number of electric field lines from the cathode end on the grid in the case of $R<R_{\min }$, which suggests that electrons drifting along the electric field lines are collected by the grid. After COMSOL completes the calculation, it generates a set of files of the electrical properties of elements and the potential of the nodes. Garfield++ has an interface for reading these files. In Garfield++, the electron transparency is defined as the ratio of the number of electrons reaching the anode to the number of electrons generated in the cathode-grid region. A condition for the maximum electron transparency can be found where all field lines bypass the grid wires.

The height of the saturation pulse in the experiment is considered to be the full penetration of electrons through the grid. Hence, the electron transparency in the experiment can be expressed as:

$T(x)=\frac{\mathrm{P}(\mathrm{x})}{P_{\max }}$,

where $P_{\max }$ is the saturation pulse height at full penetration, and $P(x)$ is the pulse height obtained under different anode voltages, as shown in Fig. 5a. The experimentally obtained electron transparency as a function of the field strength ratio $R$, together with the ones simulated with Garfield++ are plotted in Fig. 7. For different grid geometries, the simulated values almost coincide with the experimental data at various field ratios, indicating the reliability of this simulation method. Figure 8 plots the $R_{\min }$ values in the simulation versus the ones obtained experimentally, and the $R_{\min }$ values in the experiment were extracted from Fig. 7. The error bars in the experimental data stemmed from the spacing of the voltage setting. Evidently, regardless of grid parameters or 


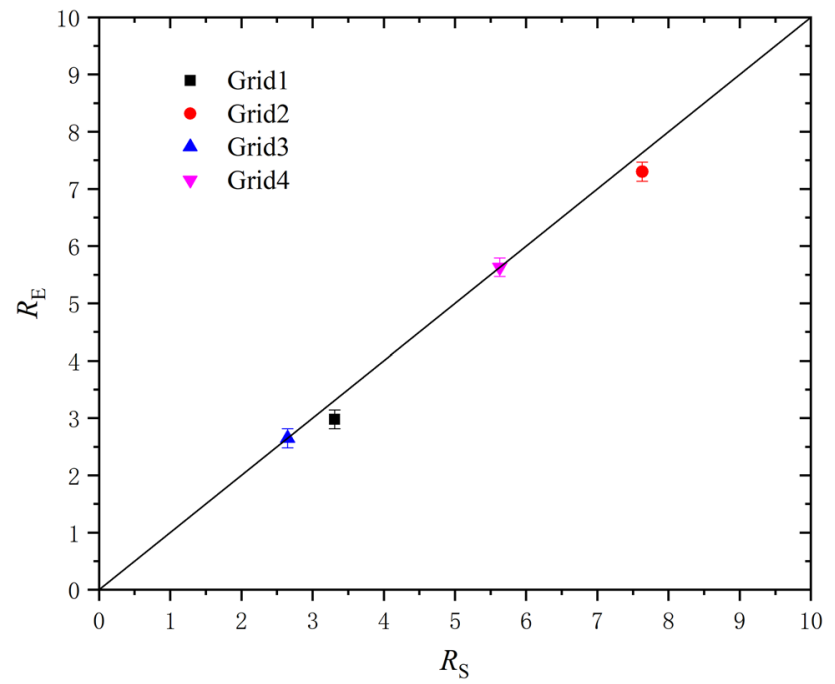

Fig. 8 Plot of the experimental $R_{E}$ versus simulated $R_{S}$ values of the minimum electric field ratio. The different symbols refer to different grids. The error bars arise from the spacing of the voltage setting in the experiment

grid geometries, the experimental and simulated value are in good agreement.

In the case of the parallel-wire grids, Grid 1 and Grid 3, the wire density parameters $\rho$ have the same value. The theoretical minimum electric field ratio $R_{\min }$ of both grids calculated by Eq. (1) was 1.926. Figure 8 shows the experimental results and simulation results, highlighting that the $R_{\min }$ values of the two grids are slightly different. As mentioned in the Introduction, the theoretical field ratio calculated using Eq. (1) is indeed lower than the experimental value. Therefore, instead of the theoretical calculation, and the simulation method can provide a better description.

When using a mesh grid as the shielding electrode, an empirical rule to determine $E_{\min }$ was suggested in Ref. [20]. According to the method given in Ref. [20], the wire density parameters $\rho_{\text {mesh }}$ were determined as $0.759 \pm 0.005$ and $0.698 \pm 0.005$, for Grid 2 and Grid 4, respectively. For application to Eq. (1), it is convenient to re-express the wire density parameters as $\rho_{\text {mesh }}=(2.417 \pm 0.005) \cdot(2 \pi r / d)$ and $\rho_{\text {mesh }}=(2.223 \pm 0.005) \cdot(2 \pi r / d)$. Both coefficients in parameter $\rho_{\text {mesh }}$ deviate significantly from 2 . Hence, for the mesh grid, multiplying the wire parameter $\rho$ in Eq. (1) by two to obtain its minimum electric field ratio $E_{\min }$ will lead to a large deviation. By comparison, the simulation method can provide more accurate results.

\section{Conclusion}

In this paper, we presented a newly built FGIC. Performing measurements with an ${ }^{241} \mathrm{Am}$ source, we observed an excel- lent energy resolution of $38 \mathrm{keV} @ 0.7 \%$ (FWHM) in 550 mbar P-10 gas when the anode signal was processed with the AMPTEK CoolFET preamplifier. For comparison, the mean chamber resolution is $46 \mathrm{keV} @ 0.85 \%$ (FWHM) using the ORTEC 142PC preamplifier under the same condition.

Different grid geometry types and grid parameters were investigated with respect to the energy resolution. The result shows that for grids with different grid parameters, as long as the respective minimum electrical field ratio is fulfilled, electrons can always be pulled down without any loss to the grid. Besides, there is a saturation limitation for the grid parameters. When the grid exhibits a small enough shielding inefficiency, the difference of the energy resolution measured by grids with different grid parameters is very small.

With sufficient wire density, both the mesh grid and the parallel-wire grid provide good electrostatic shielding, and the chamber shows similar optimal resolution. When parallel-wire grids are used as the shielding electrode, FGIC operates at a lower voltage, compared to mesh grids. However, the production of parallel wires grids requires special wire-winding equipment. Mesh grids, on the other hand, are easier to produce and commercially available. Moreover, mesh grids have good mechanical resistance. The use of mesh grids often requires higher operating voltage, and hence, their parameters should be carefully selected to avoid electron avalanche in the anode-grid region.

Since the working voltage setting of FGIC must be adjusted according to different grid parameters, it is necessary to identify a reference point to predict the appropriate voltage setting when considering the FGIC design. We applied the simulation method interfacing COMSOL and Garfield++ as an alternative to the imperfect theoretical formula derived by Bunemann et al. In our method, the minimum electric field ratio is extracted by simulating the transparency of electrons passing through the grid. For the parallel-wire and the mesh grids, the results show that the simulated value is in good agreement with the experimental value. Hence, whether utilizing a mesh or parallel wires as its shielding electrode, the proper voltage setting of FGIC can be successfully predicted via correct understanding of electron transparency through the grid using the simulation tool.

Acknowledgements This work is financially supported by the National Key R\&D Program of China (Grant No. 2018YFE0205200), the Research Program of National Major Research Instruments (Grant No. 11927901), the Natural Science Foundation of China (Grant No. 11875301, 11875302, U1867214, U1832105, U1832167), the Strategic Priority Research Program of Chinese Academy of Science (Grant No. XDB34000000), and the CAS "Light of West China" Program. This work is also supported by the Function Development Project of Chinese Academy of Sciences under Grant No. 2022G101.

Data Availability Statement This manuscript has no associated data or the data will be deposited. [Authors' comment: as for the data avail- 
ability, the only data relevant to the publication is present in form of published graphs or tables. All the datasets and figures in the paper can be available from the corresponding authors upon request].

Open Access This article is licensed under a Creative Commons Attribution 4.0 International License, which permits use, sharing, adaptation, distribution and reproduction in any medium or format, as long as you give appropriate credit to the original author(s) and the source, provide a link to the Creative Commons licence, and indicate if changes were made. The images or other third party material in this article are included in the article's Creative Commons licence, unless indicated otherwise in a credit line to the material. If material is not included in the article's Creative Commons licence and your intended use is not permitted by statutory regulation or exceeds the permitted use, you will need to obtain permission directly from the copyright holder. To view a copy of this licence, visit http://creativecomm ons.org/licenses/by/4.0/.

Funded by SCOAP ${ }^{3}$.

\section{References}

1. X.H. Zhang, S.W. Tang, P. Ma et al., A multiple sampling ionization chamber for the External Target Facility. Nucl. Instrum. Methods A. 795(21), 389-394 (2015). https://doi.org/10.1016/j.nima.2015. 06.022

2. H. Yi, Y. Zhao, W. Jiang et al., Triton identification in the ${ }^{6} \mathrm{Li}(\mathrm{n}$, t) ${ }^{4} \mathrm{He}$ reaction measurement with the grid ionization chamber at CSNS Back-n white neutron source. JINST 14(02), P02011 (2019)

3. R.O. Frisch, British Atomic Energy Report No. BR-49 (1944)

4. A. Hartmann, J. Hutsch et al., Design and performance of an ionization chamber for the measurement of low alpha-activities. Nucl. Instrum. Methods A. 814(1), 12-18 (2016). https://doi.org/ 10.1016/j.nima.2016.01.033

5. O. Bunemann, T.E. Cranshaw, J.A. Harvey, Can. J. Res. A. 27, 191 (1949)

6. W. Shockley, J. Appl. Phys. 9, 635-636 (1938)

7. S. Ramo, Proc. IRE 27, 584-585 (1939)
8. A. Göök, F.-J. Hambsch, A. Oberstedt et al., Application of the Shockley-Ramo theorem on the grid inefficiency of Frisch grid ionization chambers. Nucl. Instrum. Methods A 664(1), 289-293 (2012). https://doi.org/10.1016/j.nima.2011.10.052

9. A. Al-Adili, F.-J. Hambsch, R. Bencardino et al., Ambiguities in the grid-inefficiency correction for Frisch-Grid Ionization Chambers. Nucl. Instrum. Methods A 673(3), 116-121 (2012). https://doi.org/ 10.1016/j.nima.2011.01.088

10. H. Hara, H. Ohnuma, Y. Hoshi et al., A study of energy resolution in a gridded ionization chamber filled with tetramethylsilane and tetramethylgermanium. Structure 29(1), 1-8 (1998). https:// doi.org/10.1016/S1350-4487(97)00201-1

11. J. Ziegler, J. Biersack, M. Ziegler, SRIM - The Stopping and Range of Ion in Matter, SRIM Co. (2008)

12. A.M. Mueller, M. Döbeli, M. Suter et al., Performance of the ETH gas ionization chamber at low energy. Nucl. Instrum. Methods B 287, 94-102 (2012). https://doi.org/10.1016/j.nimb.2012.06.012

13. S.F. Biagi, Magboltz, Program To Compute Gas Transport Parameters, Version 11 (CERN, 2021)

14. https://www.amptek.com/internal-products/obsolete-products/ a250cf-coolfet-charge-sensitive-preamplifier

15. https://www.ortec-online.com/products/electronics/ preamplifiers/142pc

16. A. Gopfert, F.-J. Hambsch, H. Bax, A twin ionization chamber setup as detector for light charged particles with energies around $1 \mathrm{MeV}$ applied to the ${ }^{10} \mathrm{~B}(\mathrm{n}, \alpha)^{7} \mathrm{Li}$ reaction. Nucl. Instrum. Methods A 441(3), 438-451 (2000). https://doi.org/10. 1016/S0168-9002(99)00952-3

17. M.A. Kirsanov, N.A. Mikhanchuk, I.M. Obodovskiy et al., Eliminating the induction effect in grid ionization chambers. Instrum. Exp. Tech. 54(6), 760-764 (2011). https://doi.org/10. 1134/S0020441211050125

18. https://garfieldpp.web.cern.ch/garfieldpp/

19. COMSOL, Multiphysics (2021). https://cn.comsol.com/

20. R. Bevilacqua, A. Göök, F.-J. Hambsch et al., A procedure for the characterization of electron transmission through Frisch grids. Nucl. Instrum. Methods A 770, 64-67 (2015). https://doi.org/10. 1016/j.nima.2014.10.003 